\title{
The Smell, that Forgotten Sense
}

\author{
Ana M Koatz* \\ Psico neuro immuno biology of the Association of Allergy, (AAIBA), Argentina
}

Submission: February 28, 2017; Published: October 27, 2017

*Corresponding author: Ana M Koatz, MD specialist in Allergy and Immunology, Asthma and Immunology "Buenos Aires", Psico neuro immuno biology of the Association of Allergy, (AAIBA), Argentina, Email: akoatz@gmail.com

\section{Introduction}

Although human beings live in a constant and changing bath of smells, when it comes to defining smell, as well as describing a certain odor, the task becomes extremely difficult. The odor is described by comparison. To define the smell I will make mine the words of Marcel Proust, who says of him.

"Smell is the most personal, insubstantial, fragile and persistent of the senses, able to evoke the most vivid memories from a tiny drop of essence".

A single particle of a certain fragrance is able to open the appetite, announce danger, arouse sexual desire, evoke the most remote sensations, and recall experiences and/or emotions of the distant past. But speaking of odors, it is interesting to say that each being has the genes that code the receptors of their smell and those that code for the odors emanating from their body. The genetic variations of HCM (Major his to compatibility complex, when determining changes in the production of odorous metabolites; explain the odors associated to specific HCM types. From the above it is inferred that each individual has its characteristic odor. We think we smell with the nose, but this is like saying that we hear with the earlobe. In fact, the part of the nose that we can see from the outside serves only to receive and channel the air that contains the odoriferous molecules. The neurons that perceive these molecules, are in the depth of the nasal cavity, in a portion of cells called olfactory epithelium. Perched behind a closed curve type, on the roof of the nasal cavity, is the olfactory epithelium, which only measures a few square centimeters. It contains about 5 million olfactory neurons, in addition to their supporting cells and stem cells. In fact, there are two portions of olfactory epithelium (one on each side of the nose) that lie in a horizontal line, just below the level of the eyes (Figure 1).
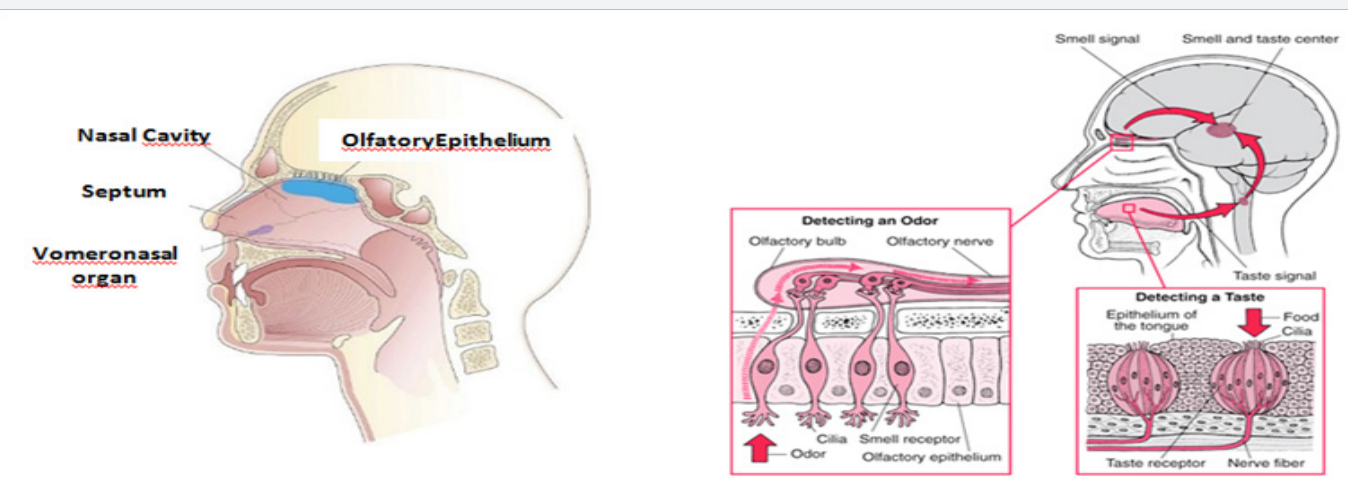

Figure 1: The olfactory pathway and its relation to the sense of taste.

Modified from Kandel ER, et al. [1] Olfactory sensory neurons are found within the olfactory epithelium in the dorsal posterior fossa of the nasal cavity, as already mentioned. These neurons project axons into the olfactory bulb of the brain, a small ovoid structure resting on the cribriform plate of the ethmoid bone [1].

\section{Structure of the Olfactory Epithelium}

There are three types of cells: olfactory sensory neurons, supporting cells and basal stem cells at the base of the epithelium. Each sensory neuron has a dendrite that projects to the epithelial surface. Many cilia protrude into the mucous layer that covers the nasal lumen. A single axon projects from each neuron to the olfactory bulb. Odorants bind to specific odorant receptors on the cilia, and initiate a cascade of events leading to the generation of action potentials in the sensory axon.

Each olfactory neuron, present in the epithelium, is covered with at least 10 cilia projecting towards a fine mucus bath, 
which is found on the cell surface. Scientists were convinced that somewhere in these cilia, there had to be receptor proteins that recognized and bonded to odoriferous molecules, thereby stimulating the cell to send signals to the brain (Figure 2).

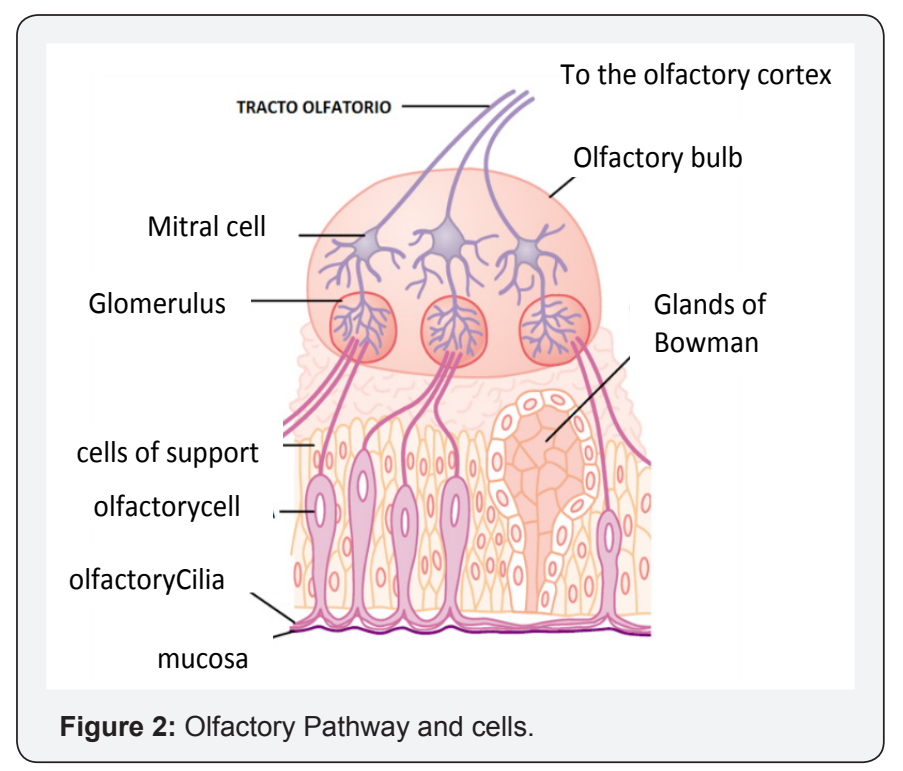

Receptor proteins would be the key to answering two basic questions about smell, explains Richard Axel [2], an HHMI researcher at Columbia University. The first question is how the system responds to thousands of molecules of different shapes and sizes, which we call odoriferous substances, "do you use a restricted number of varied receptors or a large number of relatively specific receptors?" And the second question, how does the brain use these responses to distinguish between odors?
Each olfactory neuron present in the nose has a long fiber, or axon, which is inserted through a tiny opening in the bone above it, the cribous plate of the ethmoid, to make a connection, or synapse, with other Neurons. This synapse forms, in fact, the olfactory bulb, which is a part of the brain. The olfactory bulb, a round structure like a knob, is quite large in animals with a keen sense of smell, but decreases in relative size when this ability decreases. Thus, although humans are more than twice the body size of dogs, they have a much larger olfactory bulb than the human.

\section{Olfactory Pathway}

The information is transmitted from the olfactory bulb axons of mitral and tufted transmission neurons in the lateral olfactory tract. Mitral cells project to five regions of the olfactory cortex: olfactory nucleus, olfactory tubercle, pyriform cortex, and parts of the amygdala and entorhinal cortex. Plagued cells project to the anterior olfactory nucleus and the olfactory tubercle; the mitral cells in the accessory olfactory bulb are only projected into the amygdala. The conscious discrimination of the odor depends on the neocortex (orbitofrontal and frontal crusts). The emotional aspects of olfaction are derived from limbic projections (amygdala and hypothalamus). In rodents and some mammals, a well developed vomeronasal organ is related to the perception of odors that act as pheromones; Its receptors are projected towards the accessory olfactory bulb. The olfactory pathway in the Rinencephalon is composed of the olfactory nerves, the olfactory bulbs, the olfactory belts, the olfactory striae that divide into medial, lateral, intermediate and olfactory cortex (Figure 3).

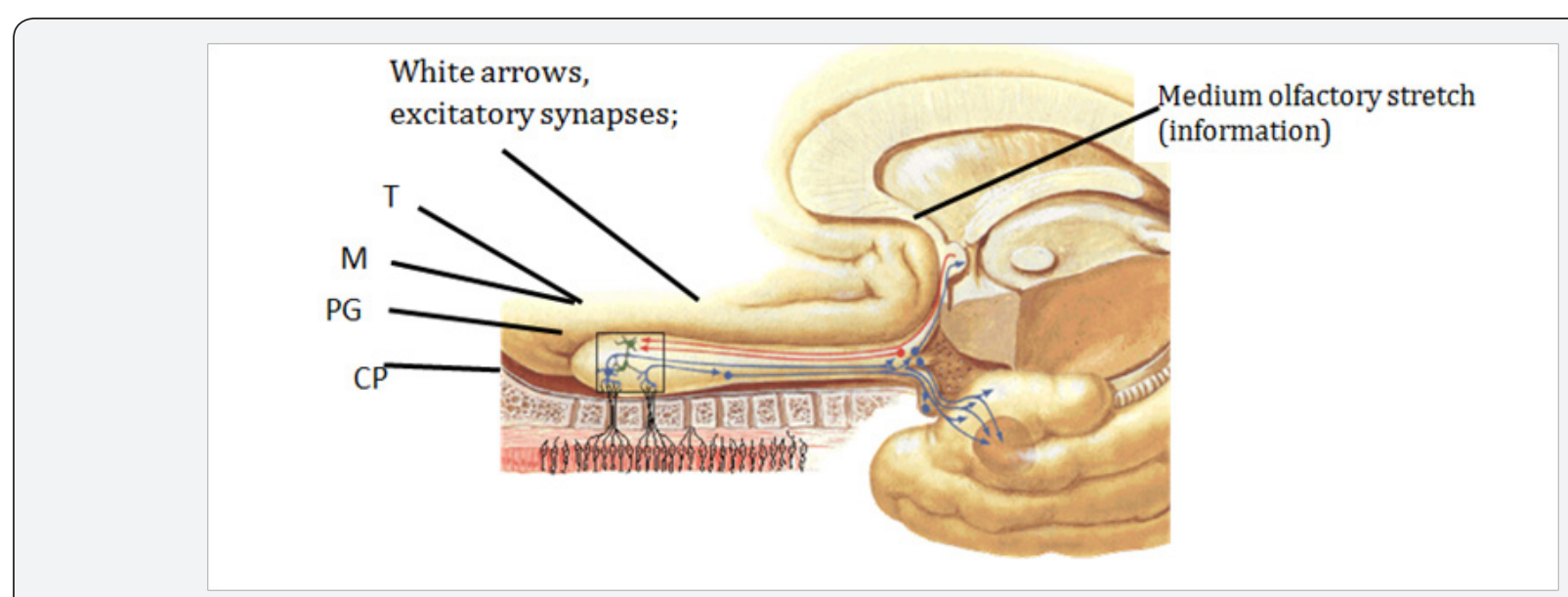

Figure 3: Olfactory pathway in the Rinencephalon.

CP: Cribriform Plate; PG: Periglomerular Cell; M: Mitral Cell; T: Plume Cell; White arrows, excitatory synapses; Black flakes, inhibitory synapses. (Adapted Mori K, Nagao H, Yoshihara Y. The olfactory bulb: Coding and processing of odor molecular information, Science, 1999; 286 (5440): 711-71

\section{Basic Neural Circuits in the Olfactory Bulb}

Olfactory receptor cells with one type of odorant receptor project into an olfactory glomerulus (OG), and olfactory receptor cells with another type of receptor project into a different olfactory glomerulus. The olfactory bulb receives the olfactory nerves and becomes thinning forming the band. The belt on reaching the perforated space above is divided into grooves. The lateral striae are projected towards the olfactory cortex. The cortex consists of the piriform cortex, periamigdaloid area and the entorhinal area. (This is the bark around the uncus). The 
medial striation is introduced at the anterior commissure and communicates both bulbs. The intermediate groove is introduced into the anterior perforated space, its function is unknown.

The rhinencephalon is in anatomical and functional relation with the limbic system. This is the neuroanatomic basis for an aroma to please us or to remind us of something or someone.

In general, the odors are composed of several molecules and each of them activates several Specific receptors. Thus a complex combinatorial code is generated which forms the socalled "odoriferous pattern" of a substance. These patterns are the basis of our ability to recognize and remember so many different odors (Figure 4).

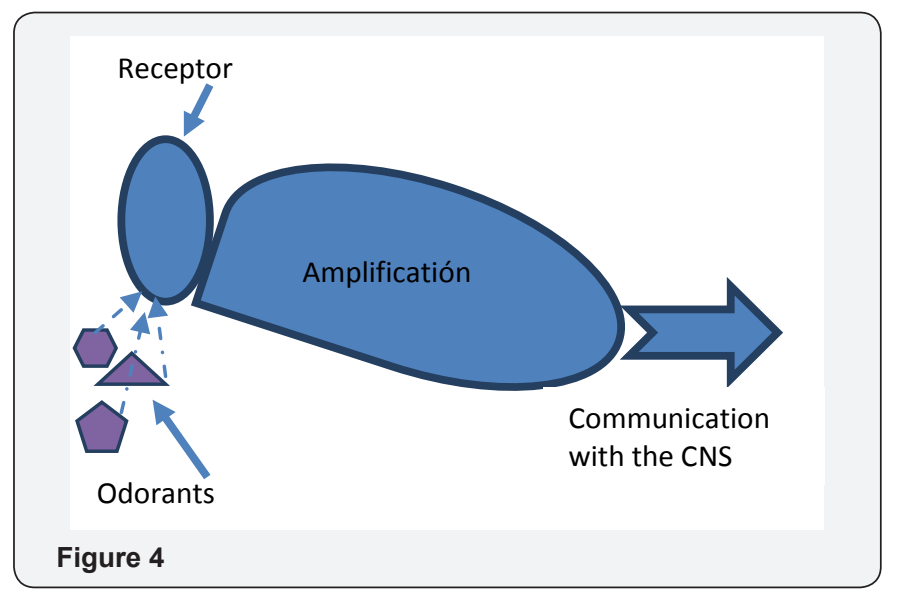

As we see for all this signal to occur the odoriferous receptors that are proteins of the cell membrane of olfactory cilia associated to protein $\mathrm{G}$ are needed. Odoriferous receptors closely resemble rhodopsin, the receptor protein present in rod-like cells of the eye. Rhodopsin and at least 40 other receptor proteins cross the cell surface seven times, giving it a characteristic snake-like shape. They also function similarly, interacting with G-proteins to transmit the signals into the cell. Because many receptors of this type share certain DNA sequences, Buck designed probes that would recognize these sequences in a group of rat DNA. He found the genes in 1991 [3].

First, they observed that the RNA obtained from rat olfactory epithelium contained a large family of genes that could range from 70 to 200. When comparing these gene products with the gene libraries (Genebank) they were found to correspond to (olfactory) genes. The second step was to sequence the proteins of some of these genes. As a result they obtained a series of proteins with 7 transmembrane domains similar to other receptors already known as the adrenergic and cholinergic receptors. They observed that in these proteins there was a great variability in the presence of amino acids, especially in domains 3,4 and 5, which could explain an enormous variability of gene control. They then demonstrated that expression of a mixture of these olfactory gene messenger RNAs occurred only in cells obtained from olfactory mucosa but not from brain, kidney, liver, heart, lung, ovary, retina or spleen. Thus, a large family of genes coded only for smell. Receptors are proteins having ligand specificity and effector specificity.

Everything said explains why we perceive as odors the various chemical substances present in the environment. The first approximation to this question consisted in supposing that on the surface of olfactory epithelial cells there had to be receptors equipped with the ability to recognize odorants. The binding of these molecules to receptors should stimulate them to send signals to the brain. As Axel relates, these receptor proteins would be the key to solving two basic dilemmas: does the system rely on a few different receptors to respond to thousands of different molecules or, conversely, are there a large number of relatively specific receptors? And, furthermore, what is the way the brain processes these responses to discriminate between odors?

Using a complex set of molecular biology techniques, Axel and Buck were able to identify in rats a large family of genes with more than 1,000 different members- that is, they represent between $2 \%$ and $3 \%$ of the total rat genes - which give rise to a similar number of proteins that are those that act as olfactory receptors. Although these receptor proteins have a fairly similar structure - they belong to the family of G proteins that cross the cell membrane - subtle differences between them are those that confer the specificity to the odoriferous molecules. Unlike the visual system that can distinguish thousands of colors using three different types of receptors, the number of olfactory receptors is comparatively huge. In humans, about 350 different types of receptors have been identified, that is, the olfactory world of a rat is infinitely richer than ours.

The other fundamental finding was the demonstration that each receptor cell exhibits, on its surface, i.e., only one type of receptor protein. Therefore, there must be at least as many cells as possible receptors. It was verified that there are about 5,000 cells that exhibit on each surface of the receptor types. In addition, it was demonstrated that each receptor has the capacity to detect a limited number of odoriferous molecules, responding to them with different intensity. In summary, each group of olfactory epithelial cells is highly specialized in detecting a few odorants.

The most astounding discovery of the team was finding that there were so many odorant receptors. The 100 different genes, which researchers first identified, were just the tip of the iceberg. It now appears that there are between 500 and 1,000 different receptor proteins, present in olfactory neurons of rat and mouse, and probably in human neurons. Says Axel. "It's 1 percent of the genome". "Most likely, the number of odorous substances far exceeds the number of receptor proteins, in a ratio of at least 10 to 1 ," says Axel. "In that case, how does the brain know what the nose smells?"

Axel began by asking how many kinds of receptor proteins are produced in a single olfactory neuron. "If an individual 
neuron expresses only a small number of receptors, or a single receptor, then the problem of determining which receptors have been activated, comes down to determining which neurons have been activated," he says. Concluded that a given olfactory neuron can produce only one or a few odoriferous receptors. (Buck and his colleagues have come to the same conclusion from their work with mice).

The next step was to find out how these odorant receptorsand the neurons that produce them-are distributed in the nose. Also, with what parts of the brain do these neurons connect? The different zones of the olfactory epithelium of the mice are shown in red, blue and yellow. A different set of genes for odoriferous receptors is expressed in each zone. When the interaction between the molecule and the specific cell occurs, it is activated by a complex cascade of chemical reactions. These have been accurately characterized by both groups and in them the formation of cyclic AMP and the subsequent opening of ion channels. This generates a signal that is the one that travels, through the thin processes of the neuroepithelial cells, to the structures of the olfactory bulb known as olfactory glomerulus.

Receptors have a 7- transmembrane structure and their activation produces, like all of their type, a cascade of intracellular events that would start with G-protein activation and increased cAMP levels (by activation of adenyl cyclase ) To produce an opening of the ion channels and an activation of the olfactory neurons.

The $\alpha$ subunit of the $G$ proteins activates the adenylate cyclase to catalyze the production of cAMP. CAMP acts as a second messenger to open cationic channels. Inward diffusion of $\mathrm{Na}+$ and $\mathrm{Ca} 2+$ causes depolarization.

When studying DNA, we could determine that there are 1000 genes that code for 1000 different olfactory receptors, each of which is expressed in millions of cells (almost every cell in the organism carries a copy of each of the genes of each being). However, the genes encoding olfactory receptor proteins are active only in olfactory neurons. Taking into account that a human contains approximately 100,000 different genes in their DNA, it can be inferred that $1 \%$ of them are dedicated to smell.

According to a paper published in Nature Genetics, the genes involved in the sense of smell are located in practice on all chromosomes and represent $1 \%$ of the entire human genome. However, the authors, from CNRS ERS of Montpellier (France), add that $70 \%$ of these genes are not functional. They are the results of a work whose objective is to determine how the olfactory genes develop and if the sense of smell in the human being has deteriorated over time as a consequence of evolution.

Using two types of genetic analysis, French scientists found that the olfactory receptor genes are located on 19 of the 23 chromosomes. Through another method they found that olfactory genes also exist in three other chromosomes. In an editorial of the magazine it is said that the human being is able to recognize more than 10,000 different odors, for which it is necessary the existence of a large number of olfactory receptors and each of them is related to a different gene. The study's authors believe that the human organism has 200 to 1,000 olfactory receptor genes. They studied 87 amino acid sequences that appear to be part of olfactory receptor genes. They found that $72 \%$ were pseudo genes, or non-functional genes because they had deteriorated as a result of mutations. "Same mutations," they wrote, "have been observed in man, chimpanzee and monkey, suggesting that many of these genetic alterations occurred during evolution in a common ancestor that may have existed between 5 and 25 million years ago." Another possibility is that some olfactory receptor genes have been "silenced" by mutation during evolution, but still exist in animals with a more developed sense of smell. It's what they try to find out with new research on animals.

In 2016, Zozulya et al. published a study in the journal Genome Biology describing that although there are more than 1,000 genes, only 347 olfaction receptor proteins are expressed. In 2003, Gilad et al. Published in Proceedings of the National Academy of Science (PNAS) that in humans more than $60 \%$ of the olfaction genes are not functional (pseudo genes), unlike the dog or the mouse in which the number of pseudo genes does not exceed the $20 \%$. The Axel and Buck groups found, in the olfactory epithelium of the nose, that neurons that produce a particular odoriferous receptor do not cluster; In contrast, these neurons are randomly distributed within certain large regions of the epithelium, called expression zones, which are symmetrical on both sides of the animal's nasal cavities. However, once the axons reach the olfactory bulb, they are rearranged so that those expressing the same receptor converge in the same place in the olfactory bulb. The result is a highly organized spatial map of information derived from different receptors. Surprisingly, the spatial map is identical in the olfactory bulbs of all the mice that were examined, says Buck. As she indicated, this information provided the key to solving an ancient enigma.

The most interesting aspect of these studies consisted in checking that recipient cells that possess the same type of conscious of almost 10,000 different receptor smells on their surface, send their processes to the same glomeruli. In this way, maintaining the specificity, the information is transmitted to other brain areas where it is combined with that from other receptors, thus generating patterns that allow the recognition. "The brain essentially says something like: I am seeing the activity in positions 1, 15 and 54 of the olfactory bulb, corresponding to the odorant receptors 1,15 and 54 , therefore, the perceived odor must be that of the jasmine, Suggests Axel.

Other odors would be identified by different combinations of this true alphabet of receptors. It is in this way that we build the memory of what we have smelled. In other words, there is a kind of labeled pathway extending from each receptor subtype to the cerebral cortex which is thus kept permanently informed about 
the degree of activation of the various receptor types. Scientists have long wondered how we can remember odors even though each olfactory neuron, present in the epithelium, survives only about 60 days, being replaced by a new cell. In most of the body, neurons die without any successor. But as olfactory neurons die, a layer of stem cells located beneath them; constantly generate new olfactory neurons to maintain a constant supply.

"The mystery was, how do we remember smells when these neurons are constantly being recycled and the new batch has to form new synapses?" Says Buck. "Now we know the answer: the memories survive because the axons of the neurons expressing the same receptor always go to the same place. Binding proteins also act as true landfills by inactivating the molecules in question, once the signal transduction has been performed, thus releasing the receptor who will be able to receive a new molecule. This DETOXIFICATION of molecules is achieved by the ligand- receptor protein phosphorylation, which is performed by two pathways. The P- 450 cytochrome mono-oxygenase pathway, which acts by hydroxylation of the substances involved and the pathway of the UDP glucoronyl transferase that catalyzes the conjugation of glucuronic acid. Olfactory perception is cataloged as an aesthetic sense, capable of producing emotions and memories, which in turn produce different thoughts and behaviors. The human vomeronasal system is the detector of "vomeroferins", substances that induce changes in maternal and sexual social behavior.

The term "vomeroferin" is preferred to refer to those substances which, by acting primarily on receptors of the vomeronasal organ of terrestrial vertebrates, especially mammals, induce changes in maternal and sexual social behavior. The nasal vomer organ (OVN) maintains connections with the hypothalamus and the limbic system, but is not known cortical representation. In the human species, stimulation of VNV with vomeroferins produces behavioral changes, along with impressive neuroendocrine changes.

Binding proteins also act as true landfills by inactivating the molecules in question, once the signal transduction has been performed, thus releasing the receptor that will be able to receive a new molecule. This DETOXIFICATION of molecules is achieved by the ligand- receptor protein phosphorylation, which is performed by two pathways. The P- 450 cytochrome mono-oxygenase pathway, which acts by hydroxylation of the substances involved and the pathway of the UDP glucoronyl transferase that catalyzes the conjugation of glucuronic acid. Olfactory perception is cataloged as an aesthetic sense, capable of producing emotions and memories, which in turn produce different thoughts and behaviors. The human vomeronasal system is the detector of "vomeroferins", substances that induce changes in maternal and sexual social behavior. The term "vomeroferin" is preferred to refer to those substances which, by acting primarily on receptors of the vomeronasal organ of terrestrial vertebrates, especially mammals, induce changes in maternal and sexual social behavior. The nasal vomer organ (OVN) maintains connections with the hypothalamus and the limbic system, but is not known cortical representation. In the human species, stimulation of VNV with vomeroferins produces behavioral changes, along with impressive neuroendocrine changes.

Given the frequency with which the otolaryngologist and the plastic surgeon surgically intervene the nose, it is essential to know the Nasal Vomer System to avoid its injury, and to know the anatomical-functional data of its activity, which confer the human Vomere-nasal system the Characteristic of a sensory system. As a corollary, and for now let's say that love comes through the smell. It is possible that the male-female attraction may be pheromonal. Pheromones are found in the secretion of the axillary glands and the dermal surface. It is possible to conclude that the sexual attraction enters the eyes, but for that "chemistry between the sexes" to occur, the responsibility corresponds to the pheromones.

\section{References}

1. Kandel ER, Schwartz JH, Jessell TM (2000) Principles of Neural Science. Principles of Neural Science ( $4^{\text {th }}$ edn). McGraw-Hill, pp. 1414.

2. Buck L, Axel R (1991) A novel multi gene family may encode odor antreceptors: a molecular basis for odor recognition. Cell 65(1): 175187.

3. Saraiva LR, Kondoh K, Ye X, Yoon KH, Hernandez M, et al. (2016) Combinatorial effects of odorants on mouse behavior. Proc Natl Acad Sci U S A 7: 113(23): E3300- E3306.
Your next submission with Juniper Publishers will reach you the below assets

- Quality Editorial service

- Swift Peer Review

- Reprints availability

- E-prints Service

- Manuscript Podcast for convenient understanding

- Global attainment for your research

- Manuscript accessibility in different formats

( Pdf, E-pub, Full Text, Audio)

- Unceasing customer service

Track the below URL for one-step submission https://juniperpublishers.com/online-submission.php 